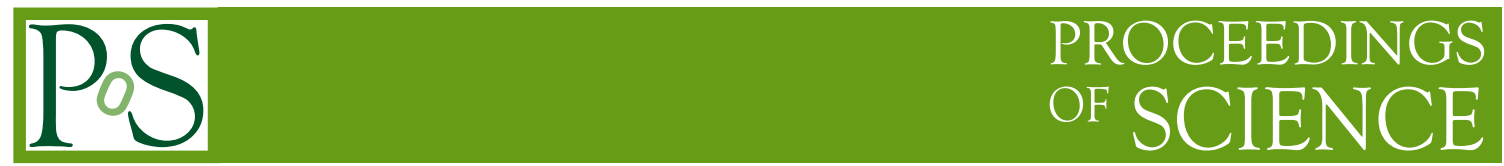

\title{
Top-quark mass measurements at the Tevatron (including Tevatron combination)
}

\author{
Cécile Deterre*t \\ Deutsches Elektronen-Synchrotron (DE) \\ E-mail: cecile.deterre@cern.ch
}

\begin{abstract}
We present a selection of top quark mass measurements from the Tevatron experiments CDF and D0. The methods developed and optimized over the years are still showing to be competitive. All the results make use of the full statistics available at the Tevatron, corresponding to an integrated luminosity ranging between 8.7 and $9.7 \mathrm{fb}^{-1}$. In particular, two recent measurements from D0 are presented: a direct mass measurement in the dilepton channel and the extraction of the pole mass from the $t \bar{t}$ cross-section measurement.
\end{abstract}

8th International Workshop on Top Quark Physics, TOP2015

14-18 September, 2015

Ischia, Italy

\footnotetext{
*Speaker.

${ }^{\dagger}$ on behalf of the CDF and D0 Collaborations
} 


\section{Introduction}

The CDF and D0 experiments at the Tevatron have performed many competitive measurements of the top quark mass over the years, and are still providing many high quality results today. The statistics of the $t \bar{t}$ sample accumulated at the Tevatron is much smaller than what is available at the LHC, but the precision of the mass measurements is limited by systematic uncertainties. In particular, the uncertainties on the jet energy scale and signal modelling are dominant in this type of measurements. We will present the different strategies chosen by CDF and D0 to constrain these uncertainties.

We present six results from CDF and D0: one using the matrix element technique, four using the template method, and one pole mass extraction from a cross-section measurement. All the measurements presented make use of the full samples recorded by the experiments which correspond to an integrated luminosity of $9.7 \mathrm{fb}^{-1}$ at D0 and between 8.7 and $9.3 \mathrm{fb}^{-1}$ at CDF.

\section{Matrix element method, principle and D0 measurement}

The matrix element method is a complex but very performant technique that was developed to measure the top mass at D0 [1]. The main principle is to compute the probability for a reconstructed event to correspond to a signal event for a given process with a given top mass $m_{t}$. Moreover, to reduce the uncertainty on the jet energy scale (JES), an in situ calibration is performed using the constant energy scale factor $k_{J E S}$ which is constrained by the invariant mass of the two jets from the $W$ boson decay to the $W$ boson mass. The probability of each event, for a given $m_{t}$ and $k_{J E S}$ is defined as:

$$
P_{s i g}=\frac{1}{\sigma_{o b s}\left(m_{t}, k_{J E S}\right)} \int d \vec{q}_{1} d \vec{q}_{2} f_{P D F}\left(\vec{q}_{1}\right) f_{P D F}\left(\vec{q}_{2}\right) \sigma\left(\vec{y}, m_{t}\right) W\left(\vec{x}, \vec{y}, k_{J E S}\right)
$$

where $f_{P D F}(\vec{q})$ is the parton density function, $\vec{q}_{1}$ and $\vec{q}_{2}$ are the four-vectors of the initial state partons, $\sigma\left(\vec{y}, m_{t}\right)$ is the leading order cross-section of the process, $\vec{y}$ represents the four-vectors of the final state partons, and $W\left(\vec{x}, \vec{y}, k_{J E S}\right)$ is a transfer function mapping the four-vectors of the final state partons $\vec{y}$ with the four-vectors of the particles reconstructed in the detector $\vec{x}$. For each event, the probability is computed for different hypotheses of $m_{t}$ and $k_{J E S}$. A likelihood function is then built by multiplying the probabilities of all events for each hypothesis. The parameters are extracted by maximizing the likelihood.

The measurement is performed in the lepton+jets channel at D0 using this technique [2,3]. The method is extended to extract the signal fraction $f$ in a step prior to the extraction of $m_{t}$ and $k_{J E S}$. After a standard selection of events containing at least 4 jets and $1 \mathrm{~b}$-tag, the sample contains around 2600 events with a purity of $66 \%$. The result is of $m_{t}=174.98 \pm 0.58$ (stat.) \pm 0.49 (syst.) GeV and $k_{J E S}=1.025 \pm 0.005$ (stat.). This is the single most precise Tevatron measurement with a relative precision of $0.43 \%$. The dominant systematics are coming from the choice of hadronization and underlying event models, as well as the residual JES uncertainty. The result in the 2D plane of $\left(m_{t}, k_{J E S}\right)$ is presented in Fig. 1. The agreement between data and expectations is checked after applying the extracted values of $k_{J E S}$ and $f$, and is shown in Fig. 2. 


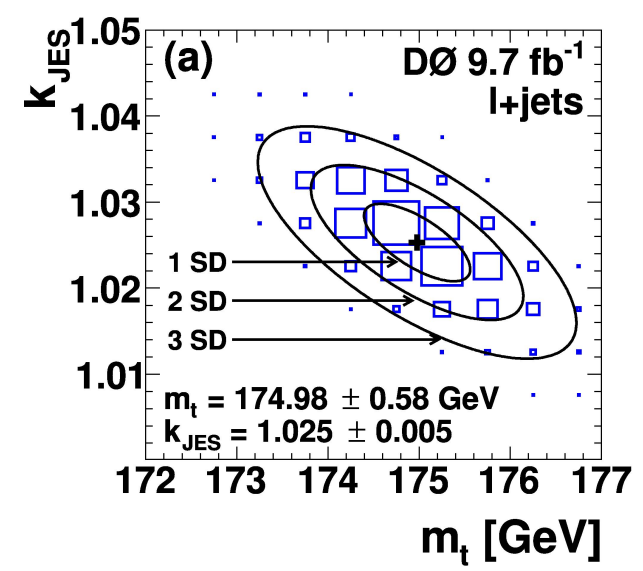

Figure 1: Result of the measurement using the matrix element technique in the $2 \mathrm{D}$ plane of $\left(m_{t}, k_{J E S}\right)[2]$.

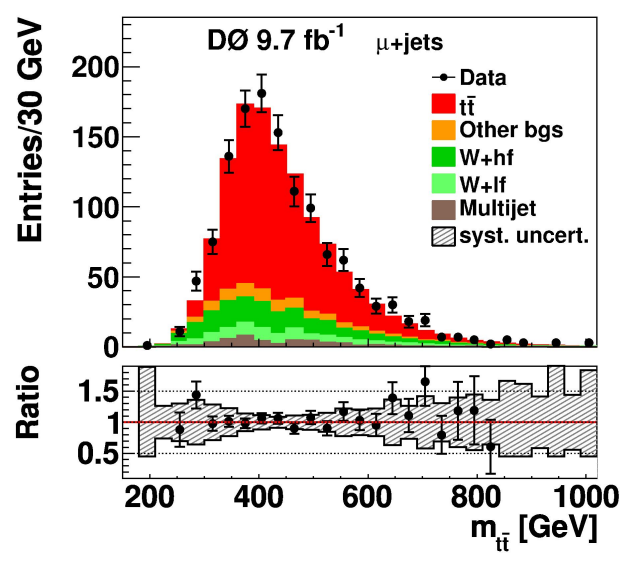

Figure 2: Agreement between data and expectations for the $t \bar{t}$ invariant mass distribution after applying the extracted values of $f$ and $k_{J E S}[3]$.

\section{Template method}

The template method relies on observables which are ideally very correlated with the top quark mass and insensitive to the systematics. The distributions of these observables are built for various mass hypotheses, and a likelihood fit is then performed to extract the value. In practice for the mass measurements, the observables are built using the decay products of the top (for instance the invariant mass of the lepton and b-jet). Since the decay products contain jets, the observable is very sensitive to the JES, and an additional observable is usually used to perform an in situ calibration and reduce the uncertainties. Another challenge is to reconstruct the top and antitop four-vectors with the final state particles and to associate properly the decays of the top to build the observable. This requires some reconstruction or pairing technique which will be described in the various analyses presented. An additional possibility is to tighten the selection by identifying more b-jets, thus reducing the possible combinations.

\subsection{Measurements at CDF}

The top mass has been measured at CDF using the template method in the three main decay channels of $t \bar{t}$ pairs: dilepton, lepton+jets and all-hadronic.

For the analysis in the dilepton channel, events with two leptons, at least two jets and large missing transverse energy $\left(\mathbb{E}_{T}\right)$ are selected [4]. Additional requirements on the invariant mass of the two leptons are applied in the same-flavour channels. Two reconstruction methods are then used. The first one called the "neutrino phi-weighting" uses all the final state particles of the event and is performant but depends a lot on the JES since it uses the jets. The most probable value of the top quark mass is extracted using this method. The second method is used to estimate the "alternative mass", defined as: $m_{l b}^{a l t}=\sqrt{\frac{\left\langle l_{1}, b_{1}\right\rangle \cdot\left\langle l_{2}, b_{2}\right\rangle}{E_{b_{1}} E_{b_{2}}}}$, where $l_{i}$ (respectively $b_{i}$ ) is the fourmomentum of the lepton $i$ (resp. b-jet $i$ ), and $E_{b_{i}}$ is the energy of the b-jet $i$. With this notation, the lepton and jet with the same index are associated to the decay of the same top quark. The 
templates are then built with a "hybrid mass" which is a linear combination of the two masses previously described, and which is optimized to reduce the total uncertainty. The result is of $m_{t}=$ $171.5 \pm 1.9$ (stat.) \pm 2.5 (syst.) $\mathrm{GeV}$, and the main uncertainties are coming from the residual JES and NLO effects.

For the measurement in the lepton+jets channel, events with one lepton, large $\mathbb{E}_{T}$ and at least four jets are considered [5]. Five channels are then defined depending on the number of identified b-jets and tight jets. The reconstruction is done by choosing the permutation with the minimal $\chi^{2}$ using the invariant masses of the reconstructed tops and $W$ bosons. Finally, three variables are used to build the templates: the values of $m_{t}$ that yield the lowest and second lowest $\chi^{2}$ values, and the invariant mass of the jets from the $W$ boson. An unbinned maximum likelihood fit is performed on the templates of the five channels (see Fig. 3). The extracted top mass is $m_{t}=$ $172.85 \pm 0.71$ (stat.) \pm 0.85 (syst.) $\mathrm{GeV}$, and the main uncertainties are coming from the residual JES and signal modelling.

The events considered for the all-hadronic channel contain at least six jets and have low $\mathbb{E}_{T}[6]$. After this preselection, the purity is of $1 / 700$ and is increased by using a neural network and b-jet identification. All permutations of jets are considered, and a $\chi^{2}$ minimization on the invariant masses of the top quark and $W$ boson is used to find the best solution. Finally, two samples are defined based on the neural network output and the $\chi^{2}$ : one to constrain the JES using the $W$ boson mass, and one to measure $m_{t}$. The extracted top mass is $m_{t}=175.07 \pm 1.19$ (stat. ${ }_{-1.58}^{+1.55}$ (syst.) GeV, and the 2D result is shown in Fig. 4.

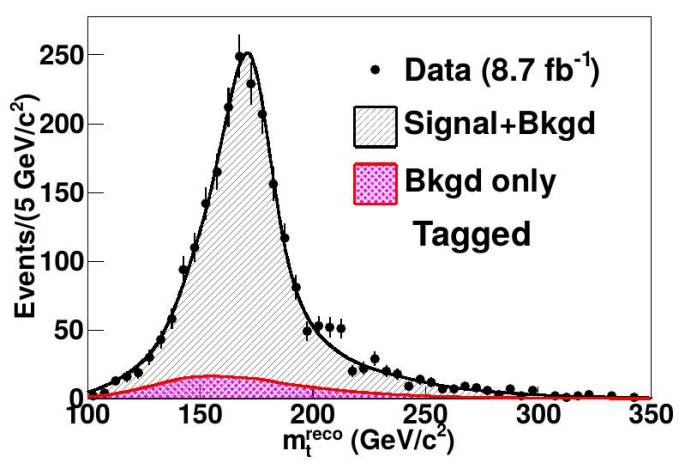

Figure 3: Example of data distribution and a template from the measurement in the lepton+jets channel [5].

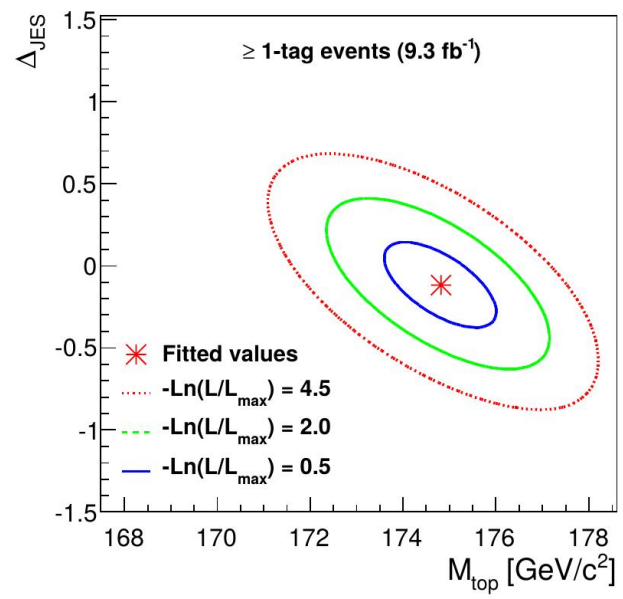

Figure 4: Result from the measurement in the all-hadronic channel in the 2D plane of $m_{t}$ and the JES scale factor [6].

\subsection{Measurement in the dilepton channel at DO}

The template method is also applied at D0 in the dilepton channel [7]. Events with two leptons and at least two jets, of which one has to be identified as a b-jet, are selected. The JES is constrained using the lepton+jets measurement described in Sec. 1. The reconstruction of the top kinematics is performed using the "neutrino weighting" method [8]. Various hypotheses on the 
neutrino rapidities are made, the kinematic equations are solved and a weight is computed to account for the compatibility of the computed neutrino momenta with the measured $\mathbb{E}_{T}$. For each event, a distribution of weights is obtained as a function of the mass hypothesis. The mean and standard deviation of this distribution are used to build the templates (see Fig. 5). The result of the template fit is $m_{t}=173.32 \pm 1.36$ (stat.) \pm 0.85 (syst.) GeV. This is the most precise dilepton measurement at the Tevatron with a relative uncertainty of $0.93 \%$.

\section{Pole mass extraction at D0}

The measurements presented so far are calibrated with the Monte Carlo simulation. The top quark mass in simulation is however not theoretically well defined. A determination of the pole mass is possible from the $t \bar{t}$ cross-section measurement. The results are not as precise as the direct measurements, but don't suffer from the ambiguity of the definition of the top quark mass.

This analysis uses a combined measurement of the production cross-section in the dilepton and lepton+jets channels using multivariate techniques and template fits [9]. The cross-section is measured for various hypotheses on the top mass. The dependence of the measurement and the predictions on $m_{t}$ are parameterized and the mass is extracted using a normalized joint likelihood function. The result is $m_{t}=169.5_{-3.4}^{+3.3}$ (total) $\mathrm{GeV}$ and is shown in Fig. 6. With a relative uncertainty of $2 \%$, this is the most precise pole mass measurement at the Tevatron.

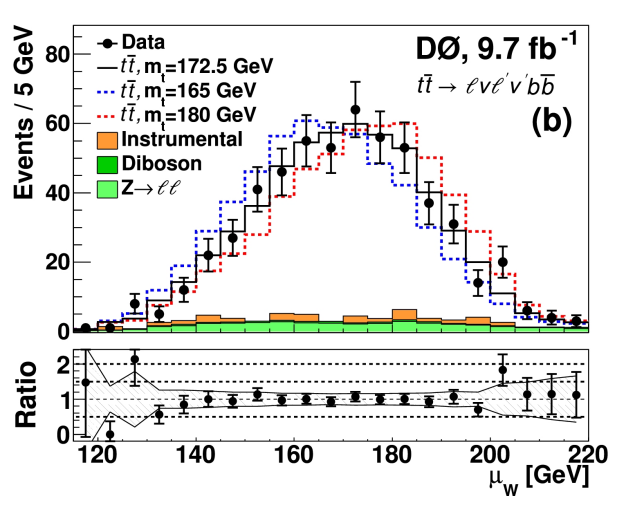

Figure 5: Distribution in data compared to templates used for the D0 top quark mass measurement in the dilepton channel [7].

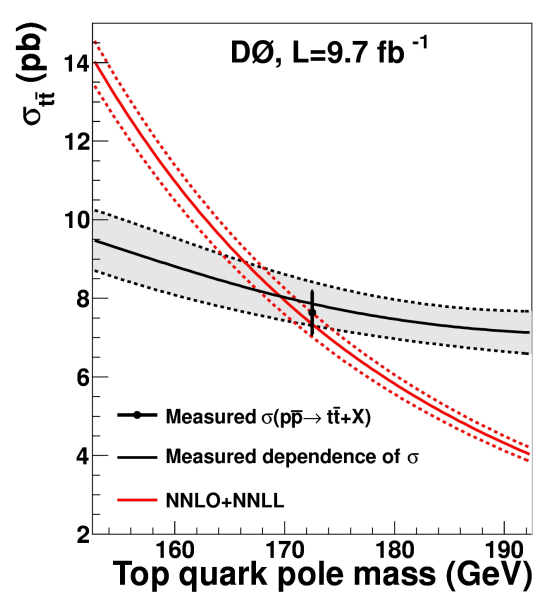

Figure 6: Result of the pole mass extraction from the $t \bar{t}$ cross-section measurement at D0 [9].

\section{Tevatron combination}

Several combinations of the Tevatron results have been performed over the years, requiring careful studies of the correlations between the systematic uncertainties across the various channels, measurements and experiments. The latest combination allows to reach a relative precision of $0.37 \%$ on the mass with a central value of $m_{t}=174.34 \pm 0.37$ (stat.) \pm 0.52 (syst.) GeV [10]. The 
two measurements in the dilepton channels from CDF and D0 presented here are not used for this combination.

\section{Conclusion}

In conclusion, the Tevatron experiments have developed several techniques to measure the top quark mass that are also used at the LHC experiments on larger samples. The results are however still competitive and were the most precise until very recently.

\section{Acknowledgments}

Thanks to my collaborators from CDF and D0 for their help in preparing the presentation and this article. I also thank the staffs at Fermilab and collaborating institutions, and acknowledge the support from the Helmholtz association.

\section{References}

[1] The D0 Collaboration, A precision measurement of the mass of the top quark, Nature 429, 638-642 (2004)

[2] The D0 Collaboration, Precision Measurement of the Top Quark Mass in Lepton+Jets Final States, Phys. Rev. Lett. 113, 032002 (2014)

[3] The D0 Collaboration, Precision measurement of the top-quark mass in lepton+jets final states, Phys. Rev. D 91, 112003 (2015)

[4] Measurement of the top-quark mass in the t $\bar{t}$ dilepton channel using the full CDF Run II data set, Phys. Rev. D 92032003 (2015)

[5] The CDF Collaboration, Precision Top-Quark Mass Measurement at CDF, Phys. Rev. Lett. 109, 152003 (2012)

[6] The CDF Collaboration, Measurement of the top-quark mass in the all-hadronic channel using the full CDF data set, Phys. Rev. D 90, 091101(R) (2014)

[7] The D0 Collaboration, Precise measurement of the top quark mass in dilepton decays using optimized neutrino weighting, submitted to Phys. Lett. B, arXiv:1508.03322 (2015)

[8] The D0 Collaboration, Measurement of the Top Quark Mass Using Dilepton Events, Phys. Rev. Lett. 80,2063 (1998)

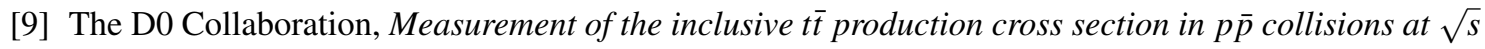
$=1.96 \mathrm{TeV}$, Conference note 6453 (2015)

[10] The CDF and D0 Collaborations, Combination of CDF and DO results on the mass of the top quark using up to $9.7 \mathrm{fb}^{1}$ at the Tevatron, arXiv:1407.2682 (2014) 